\section{Revisitando a}

\section{antropofagia: os}

estudos culturais

brasileiros nos

\section{anos 90}

\section{RESUMO}

Este ensaio procura situar as correntes principais de apreensão de fenômenos culturais na década de 90: uma, herdeira dos estudos sociológicos de Antonio Candido e da Teoria Crítica praticada por Roberto Schwarz, onde a História entra como peça fundamental e 0 aporte marxista serve como ponto de partida para uma revisão contemporânea. Uma outra que, utilizando os avanços formalistas e a autoridade científica conferidos por abordagens estruturais, retoma as vozes dos fundadores de uma "semiótica brasileira", Haroldo de Campos, Augusto de Campos e Décio Pignatari. E a problematização do conceito de entrelugar (elaborado por Silviano Santiago no final dos anos 70) que vai representar uma das saídas mais consistentes para 0 eterno dilema entre formalismos e sociologismos da cultura brasileira.

\section{ABSTRACT}

This essay presents a mapping of contemporary cultural critique in Brazil with its main approaches: one by the heirs of Antonio Candido and the Critical theory represented by Roberto Schwarz, in which history and Marxian tradition are articulated in a contemporary revision. Another perspective given by the structuralist and semiotic tradition that recovers founders like Haroldo de Campos, Augusto de Campos and Décio Pignatari. And a third one which develops the notion of the entrelugar (the in-between) as defined by Silviano Santiago by the end of the seventies, which is one of the most consistent alternatives to the always present opposition between "formalisms" and "sociologisms" in Brazilian culture. Keywords Brazil, Critical theory, identity, Cultural Studies.

\section{PALAVRAS-CHAVE (KEY-WORDS)}

- Brasil

- Teoria crítica (Critical Theory)

- Identidade e Estudos Culturais (Identity and Cultural Studies)

\section{Angela Prysthon}

Profa. Dra. PPG Comunicação da UFPe
No BRAsil da dÉCAdA dE 80, o ethos dominante envolve o pós-moderno, grande parte dos conceitos que 0 circundam, e as nuances mais intensas de um cosmopolitismo mais tradicional. Como se obedecendo à ondulação de um pêndulo, a década de 90 assiste à reemergência da busca da identidade nacional, a retomada da "essência brasileira" nas artes e cultura. Todas as tendências e políticas culturais mundiais originadas mais ao final dos anos 80 , como o multiculturalismo, o politicamente correto, a world culture, contribuíram para essa reorientação cultural. A crítica da cultura brasileira contemporânea vai sendo influenciada pelos Estudos Culturais e pelas teorias pós-coloniais contemporâneos europeus e norte-americanos, e também redimensionada pelo quase súbito e crescente prestígio internacional e nacional que gozam certos nomes de uma arraigada tradição da teoria brasileira como Roberto Schwarz, Silviano Santiago, Antonio Candido, Heloísa Buarque de Holanda e Renato Ortiz, entre outros.

Tais fatores vão ser cardeais no sentido de fazerem reaparecer com força noções que andavam parcialmente adormecidas durante toda uma década. Grande parte da teoria cultural elaborada a partir do final dos anos 80 vai ser baseada nessas noções ou abordagens. A premissa elaborada por Schwarz, por exemplo, em Ao Vencedor as batatas (1977), sobre "as idéias fora do lugar" na sociedade brasileira - a saber, o ensaio específico sobre o liberalismo adotado no Brasil do século XIX e usado como discurso de um regime escravocrata - é retomada no Brasil e no 
exterior como ponto de partida para uma crítica marxista do aporte celebratório da diferença e para o redimensionamento do conceito de dependência:

Schwarz, that is, thinks of the "national" as a product - social, economic, histo-rical - of the international, the global. The part is thought through the whole. "Dependency", it now becomes apparent, could never, despite its formal grasp of imperialism and colonialism as global and systematic, do otherwise than think the whole through the part, the international through the national. Those who currently protest the anachronism of the "national" as such, reducing all to a question of the "transcultural" and global hybridity, merely think the whole without the part, apparently "solving" the problem by conceptual fiat but in fact condemning themselves to theoretical and political irrelevance. (LARSEN, 1995, 214-5)

Schwarz é o mais relevante dos críticos para a continuidade - ou talvez até constituição - de uma teoria crítica no pensamento brasileiro. Tal teoria está baseada na constante vigilância contra as armadilhas da inversão do binarismo cópia/original, contra o elogio do "atraso", contra, enfim, um sentido de "nacional por subtração":

Quem diz cópia pensa nalgum original, que tem a precedência, está noutra parte, e do qual a primeira é o reflexo inferior. Esta diminuição genérica freqüentemente responde à consciência que têm de si as elites latino-americanas, (...). Nem por isso adianta passar ao pólo oposto: as objeções filosóficas ao conceito de originalidade levam a considerar inexistente um problema efetivo, que seria absurdo desconhecer. A historiografia da cultura ficou devendo o passo glo-balizante dado pela economia e sociologia de esquerda, que estudam o nosso "atraso" como parte da história contemporânea do capital e de seus avanços. (SCHWARZ, 1987, 47-8)

Se Schwarz vai ser recuperado nos anos 90 como a expressão máxima de um marxismo renovado e da teoria crítica contemporânea no Brasil, seu antecessor (e podemos arriscar até mentor) Antonio Candido é visto não apenas como o marco zero de uma sociologia da cultura brasileira, mas também como o antídoto para os excessos do estruturalismo e pósestruturalismo que haviam se instalado no país desde os anos 60, e passa, inclusive, a ser apontado em algumas esferas como precursor dos estudos subalternos contemporâneos (mais ou menos num mesmo sentido em que Raymond Williams foi recuperado de forma geral na América Latina ${ }^{1}$ ). Seus textos sobre a literatura regional e as relações entre subdesenvolvimento e literatura marcaram toda uma geração de críticos brasileiros, tanto os que adotam, como os que rejeitaram veementemente a abordagem sociológica da cultura. Para Candido, a consciência do subdesenvolvimento econômico e da dependência cultural é pré-requisito fundamental para uma possível superação deles, especialmente desta última. Tal discussão vai servir como perspectiva inicial para a ligação feita por Alberto Moreiras da sociologia cultural de Candido com o grupo de estudos subalternos latino-americanos:

En mi opinión esta afirmada "normalidad" o "naturalidad" de la dependencia cultural se convierte en el asunto decisivo y en la piedra de toque contra la que el subalternismo latinoamericano puede y debe organizar su deseo de oponerse a paradig-mas previamente articulados. 
(MO-REIRAS, 1996, 881)

Antonio Candido é um dos poucos críticos latino-americanos a comentar e procurar as razões para a distância e a falta de intercâmbio entre os diversos países que compõem a América Latina e particularmente entre os dois blocos lingüísticos majoritários, as Américas espanhola e portuguesa. Com uma obra que se destaca no pensamento brasileiro desde os anos 40, Candido vem recolocando ao longo das décadas - papel da literatura na construção da "nacionalidade", as questões sobre cópia e original, a dialética do cosmopolitismo brasileiro (mesmo que indiretamente), a existência de uma latino-americanidade e as definições de subdesenvolvimento.

Considerada como derivação do atraso e da falta de desenvolvimento econômico, a dependência tem outros aspectos que manifestam a sua repercussão na literatura. Lembremos de novo o fenômeno da ambivalência, traduzido por impulsos de cópia e rejeição, aparentemente contraditórios quando vistos em si, mas que podem ser complementares se forem encarados desse ângulo (CANDIDO, 1989, 156).

Em 1998, ano em que Candido completa oitenta anos de vida, sai um suplemento Mais! da Folha de São Paulo em sua homenagem. Nele reúnem-se desde usuais admiradores e continuadores da sua sociologia crítica (e crítica sociológica) - Walnice Nogueira Galvão, Luiz Costa Lima, Maria Sylvia Carvalho Franco - até dois dos mais renomados teóricos da linguagem literária no Brasil- Haroldo de Campos e Leyla Perrone-Moisés. Essa reunião em torno de Candido dá uma medida aproximada da grande fluidez teórica entre "formalismos", "historicismos" e "sociologismos" na década (de certo modo, demonstrando a continuidade do processo iniciado nos anos $80 \mathrm{com}$ a nova história brasileira e uma maior autocrítica por parte do estruturalismo).

Tanto Candido como Schwarz são extremamente influentes no meio acadêmico brasileiro, com vários nomes de peso tendo sido formados nessa tradição. Cabe destacar o trabalho de Flora Süssekind, que nas suas meticulosas análises literárias, tenta apreender, ao lado de uma abordagem estilística geral, as implicações do discurso social das épocas que toma como objeto de estudo. Nesse sentido, podem ser consideradas a concepção sociologizante da tradição de Candido e Schwarz e também uma dedicada percepção histórica, como demonstram seus estudos mais longos desde o final dos anos 80, Cinematógrafo de Letras (1987), sobre as relações entre técnica e a literatura pré-modernista brasileira, e O Brasil não é longe daqui (1990), sobre os narradores-viajantes nas décadas de 1830 e $1840 . \quad$ Outro nome que vem trabalhando no sentido de dar continuidade à essa abordagem históricosocial da literatura - sem perder de vista a dimensão estrutural, é óbvio- é Francisco Foot Hardman, que relaciona concepções de progresso, modernidade, ideologia e política ao discurso do trabalho, do anarquismo e da literatura no Brasil do final do século XIX e início do século $X X$, em Nem pátria, nem patrão (1985) e Trem fantasma. A modernidade na selva (1988).

Essa nova concepção brasileira de discurso acadêmico -que tenta fronteiras mais fluidas entre análise literária, sociologia da cultura e discurso historiográfico - vai ser o lado mais bemsucedido -embora nada disso seja muito inédito ou propriamente pós-moderno- da "pós-modernização" da cultura brasileira. Mesmo alguns dos mais estabelecidos e tradicionais professores de literatura, como Alfredo Bosi, experimentaram essa fusão da sociologia literária à maneira de Candido, de uma historiografia mais aberta e da análise formal. Em Dialética da colonização (1992), Bosi desvela 
as implicações da experiência colonial brasileira através não só de uma história e uma literatura específicas e os comentários sobre essas, mas as relações entre elas na contemporaneidade, num exercício constante de diálogo entre o passado, o presente e o futuro brasileiros - sempre em relação a um contexto universal:

É então que imagens míticas de outros tempos se atualizam na memória das culturas tentando fazer justiça à densidade sempre nova da condição humana. No caso da formação colonial brasileira, essas transferências simbólicas, que varam tempos e lugares, operam com experiências sociais peculiares à nossa história: mas, enquanto modos de produzir significados, elas confirmam uma constante do processo de aculturação tal como o conhecemos desde, pelo menos, a antigüidade oriental e mediterrânea. (BOSI, 1992, 383)

É notável, contudo, a ausência das alusões diretas ao pós-modernismo ou a pós-modernidade no discurso teórico brasileiro contemporâneo. Uma das exceções é Sergio Paulo Rouanet, que na maioria dos ensaios de Mal-estar na modernidade (1993), retoma o conceito de pós-moderno (ou, mais precisamente, a sua negação) na década de 90 - na década anterior havia sido um dos principais "divulgadores" do conceito no país - para novamente especular sobre as condições da crise e da continuidade da modernidade no mundo e na periferia. Retomando o tema de um lluminismo renovado em "A coruja e o sambódromo", seu principal ataque é ao relativismo cultural e aos "etnocentrismos" que parecem ter invadido todas as esferas teóricas nos últimos anos.

O iluminista latino-americano combate o eurocentrismo, porque é a extra-polação abusiva de uma particularidade que se quer hegemônica. Mas combate também o latinocentrismo, forma equivocada de responder a um particularismo com outro particu-larismo. Nesse combate, ele pode apoiar-se na própria tradição, a tradição das Luzes, época universalista e portanto antietnocêntrica por excelência. (ROUANET, 1993, 90)

Entretanto, passado o gosto pela polêmica das primeiras encarnações do conceito de pós-moderno no país, o problema parece ser agora definir em que medida os conceitos relativos à ou modificados pela pós-modernidade (e em menor escala, mais fechado na análise de cada gênero artístico e certos estilos, ao e pelo pós-modernismo) determinam as condições da modernidade brasileira e o papel do país em relação ao desenvolvimento e aos desafios propostos por essa nova acepção de globalização surgida nos anos 90. Nessa linha, destaca-se a obra de Renato Ortiz, sociólogo e teórico da comunicação, que vem refletindo desde os anos 80 sobre como a modernidade e os discursos que a caracterizam - principalmente os discursos relativos à cultura de massas - vêm se acomodando no Brasil (sem contar com o trabalho sobre os inícios da modernidade na Europa e, particularmente, na França - 1991). Primeiro com Cultura brasileira e identidade nacional (1985), no qual faz um apanhado das principais idéias que embasaram a cultura do país, desde a ideologia do branqueamento do século XIX até as utopias revolucionárias da década de 60. Depois com A moderna tradição brasileira (1988), em que relata a emergência de uma indústria cultural no país. Mas é com Mundialização e cultura (1994) que ele problematiza as questões relativas à identidade nacional tendo em vista as transformações e complexidades trazidas à tona pela globalização (entendida como desdobramento transnacional do 
capitalismo tardio).

Refletir sobre a mundialização da cultura é de alguma maneira se contrapor, mesmo que não seja de forma absoluta, à idéia de cultura nacional. Diante deste desafio, temos às vezes a tendência em negar o processo que estamos vivendo, nos refugiando nas certezas e convicções contidas nas análises clássicas das Ciências Sociais. (ORTIZ, 1994,116)

Como a literatura, a teoria literária e a sociologia da cultura, outra área de estudos que se beneficia de um viés múltiplo e mais aberto (e talvez mais relativista, como teme Rouanet) de análise no Brasil do final da década de 80 e início da década de 90 é a história. Há uma preocupação com a devida representação das manifestações culturais (literatura, particularmente) não apenas como mera apresentação de documentos, mas como partícipe e agente da história. O caso evidente de Nicolau Sevcenko demonstra as possibilidades da Nova História associada à multidisciplinaridade dos Estudos Culturais. Seu livro Orfeu extático na metrópole: São Paulo, sociedade e cultura nos frementes anos 20 (1992) combina expressão literária, apreciação minuciosa dos arquivos da época e o mapeamento do imaginário popular coletivo para delinear conceitos de modernidade e os efeitos dessa modernidade na história brasileira -com uma concentração maior, naturalmente, na história da cidade de São Paulo.

Ocupando uma trincheira quase oposta a essa interpretação mais social e histórica da cultura e do discurso literário estão membros igualmente destacados dos Estudos Culturais brasileiros. Como se a história cultural brasileira estivesse sempre marcada por essa oposição entre análise histórica e social e análise formalista e estetizante. O que é sugerido através da polêmica ocorrida em meados dos anos 80 entre Roberto Schwarz e Augusto de
Campos sobre o poema póstudo, escrito por este último, onde foram discutidas questões como pós-moderno, formalismos, conteudismos. Tal polêmica caracterizaria um confronto permanente na teoria literária brasileira que, segundo José Miguel Wisnik, teria duas leituras possíveis:

Nós teríamos de fato, na polêmica entre Roberto Schwarz e Augusto de Campos, um confronto entre uma leitura literária que privilegia a prosa e outra que privilegia a poesia. Uma que privilegia o vínculo da História social interna à obra e outra que, de certo modo, descaracteriza esse vínculo como sendo importante e se interessa pelo diálogo sincrônico da obra literária, pelo permanente diálogo entre as obras, o que se faria como um modo de reler o passado, recriar o passado e o futuro no permamente presente da linguagem (WISNIK, 1988, 258).

Essa segunda facção da crítica cultural brasileira caracteriza-se também por uma retomada das teorias antropofágicas sugeridas na obra de Oswald de Andrade e a adoção da semiótica geral como metodologia possível. Seus mais famosos -e antigos - membros incluem os poetas e teóricos Augusto e Haroldo de Campos e Décio Pignatari, ativos propagadores da semiótica desde os anos 50 e 60.

Entretanto, o que interessa nessa versão pós-moderna, fim-de-secular, de paradigmas teóricos da modernidade e de estratégias estilísticas do modernismo, mais do que o conjunto de obras teóricas e críticas desses autores especificamente, é a adaptação de seus esquemas de pensamento, de suas teorias da tradução, da sua estética literária baseada na idéia de canibalismo cultural tirada do Manifesto Antropofágico de Oswald de Andrade para o campo da crítica cultural contemporânea, dos Estudos Culturais propriamente ditos e de uma emergente teoria pós-colonial brasileira, como sugere, por exemplo, Else Vieira:

The perspective I would like to fore- 
ground is that the Orient-imported label, by reintroducing a heightened awareness of a (post)colonial condition, brings to visibility a Latin American body of postcolonial theory and even enables its constitution as such. (...) In the early 1920s, Oswald de Andrade and Mário de Andrade, associated with the movement of anthropophagy (can-nibalism) against mental colonialism, also emerge as postcolonial thinkers. (VIEIRA, 1999, 274)

A Antropofagia cultural, tal como proposta por Oswald e mais ainda por uma interpretação que talvez pudéssemos chamar de semiótica, seria a condutora da diferença brasileira e, assim, serviria como estatuto para a constituição de um ponto de vista pós-colonial para a teoria brasileira e para a superação das noções de atraso e descompasso.

But the main point is that cannibalism or the mask or getting into another's skin are diverse ways of describing a relation not grounded on binary power oppositions (superior-inferior) but on the notion of continuation and becoming, a becoming that operates at threshold of "fusion and distinction", permanence and transcendence, union collateral with autonomy (sic), etc. (VIEIRA, 1996, 11)

Nem sempre utilizando uma perspectiva lingüística ou semiótica à maneira dos irmãos Campos ou Pignatari, os "canibalistas" pós-modernos, entretanto, propõem a identidade nacional como uma construção discursiva que visa a delinear diferenças, inverter e subverter oposições e questionar a dependência cultural. $\mathrm{O}$ modernismo - especialmente as duas figuras-chave, Oswald de Andrade e Mário de Andrade - vai sendo viabilizado, através das interpretações contemporâneas, como o discurso que primeiro enuncia a relação de alteridade como constitutiva da formação da cultura brasileira. Os manifestos de Oswald são tomados como pontos de partida para a constituição de uma teoria da cultura "nativa":

Os Manifestos dão conta da ausência de teorias explicativas da realidade local e, mais, da explicação sempre enviesada pelas teorias cosmopolitas espelhadas no liberalismo econômico e social. Propondo assim uma via alternativa de análise dos modelos sociais, crítico às mais recentes pesquisas desenvolvidas no campo do pensamento europeu da época. (VERONA, 1996, 47)

O Manifesto Antropofágico de Oswald de Andrade torna-se, assim, para esse viés de interpretação, um dos textos fundamentais de uma teoria "brasileira" da cultura, uma espécie de atestado de uma "precoce" pós-modernidade latinoamericana, quase como a precondição de existência de Estudos Culturais brasileiros, uma espécie de pedra de toque de uma abordagem pós-colonial no país.

Fazendo uma pilhagem filosófica em Freud, Marx, Kierkegaard, Nietzsche, Schopenhauer e Engels, Oswald de Andrade vai propor que a força vital do homem é a devoração. Ou seja, ela é o impulso necessário para a criação e crítica de um projeto cultural e ideológico que questione a dependência e a colonização. (...) Assim, pela antropofagia como perda produtiva, articulada à paródia e à inversão hierárquica carnavalizante, Oswald de Andrade reinstala uma modalidade de prática sacrificial (HELENA, 1996, 62).

Fundamentando, pois, a subversão (canibal) parodística como o principal elemento de desestruturação da hegemonia ocidental, a antropofagia como teoria estética instaura simultaneamente o lugar da tradição num discurso que se pretende de ruptura (o discurso da modernidade). O que essa condição paradoxal provoca 
é precisamente as duas linhas de interpretação do canibalismo cultural brasileiro: a primeira que vê a antropofagia como sinal de uma modernidade acrítica e a outra que conclui da deglutição (que pode ser associada a um estado híbrido, já que se devora o Outro, digere-se o Outro, tornando-se um pouco esse Outro) o espaço pós-moderno avant la lettre na América Latina.

Repete-se um pouco a tensão entre os dois pólos da crítica de cultura brasileira, o da linha sociológica e o do da linha estruturalista. Renato Ortiz, por exemplo, através do instrumental fornecido pela teoria crítica frankfurtiana, detecta na modernidade brasileira um sentido de acomodação:

Moderno como tradição, mas não como colocava Octavio Paz, enquanto tradição de ruptura; os sinais da "modernidade" brasileira indicam que realmente "somos", e que por isso não devemos nos rebelar na direção de um outro futuro.(ORTIZ, 1988, 20910)

Um dos mais influentes críticos brasileiros contemporâneos, Silviano Santiago, por sua vez, propõe o modernismo brasileiro (e particularmente o modernismo de Oswald de Andrade) como recuperação suplementar da tradição européia, como discurso utópico do "eterno retorno em diferença" (SANTIAGO, 1989, 109), como possiblidade de repensar as vanguardas em relação à tradição (fazendo desse modo uma ponte com o pós-moderno através do discurso modernista), como ponto de partida para a constituição de um pensamento pós-colonial que desconstrua a história da dependência:

O sentido da paródia em Oswald de Andrade é você comer o outro para ser mais forte. O pensamento dele está muito vinculado, a meu ver, a uma discussão sobre dependência cultural. É uma maneira do Brasil se afirmar pela via oposta à da colonização. (IDEM, IBIDEM, 121-2)

A discussão sobre dependência cultural vai ser, aliás, um dos pontos de partida para que Silviano Santiago, baseado numa abordagem pós-estruturalista e inspirado pela desconstrução derrideana, elabore a sua definição de entrelugar, primeiramente no ensaio escrito em 1969, mas estendida até os anos 90, quando vai ser recorrente não só no trabalho do próprio Santiago, mas principalmente em inúmeros outros teóricos brasileiros. Em "O entre-lugar ${ }^{2}$ do discurso latino-americano", Silviano Santiago lança mão da história colonial e das relações entre indígenas e catequizadores para chegar a uma proposição talvez por demais generalizante para a contemporaneidade cultural da América Latina.

Entre o sacrifício e o jogo, entre a prisão e a transgressão, entre a submissão ao código e a agressão, entre a obediência e a rebelião entre a assimilação e a expressão, - ali, nesse lugar aparentemente vazio, seu templo e seu lugar de clandestinidade, ali se realiza o ritual antropofágico da literatura latino-americana. (IDEM, 1978, 108)

O entrelugar explicaria fundamentalmente a diferença subalterna como devendo ao mesmo tempo às idéias de progresso e modernidade - cumprindo uma espécie de pacto com a História ocidental - e à incorporação de elementos alternativos das minorias lingüísticas, sociais e culturais que compõem os tempos e espaços multifacetados das culturas da América Latina. Com essa mescla de culturas e imaginários, a América Latina representaria desde o período de colonização não somente esses processos de hibridização em si, mas a consciência deles: 
A maior contribuição da América Latina para a cultura ocidental vem da destruição sistemática dos conceitos de unidade e de pureza: estes dois conceitos perdem o contorno exato do seu significado, perdem o seu peso esmagador, seu sinal de superioridade cultural, à medida que o trabalho de contaminação dos latino-americanos se afirma, se mostra mais e mais eficaz. (IDEM, IBIDEM, 18)

A sua proposta, contudo, revela-se aplicável não só à cultura latino-americana, mas ao que ele chama de "cultura dominada" em geral; constituindo então a desconstrução da hierarquia colonizadorcolonizado.

Paradoxalmente, o texto descolonizado (frisemos) da cultura dominada acaba por ser o mais rico (não do ponto de vista de uma estreita economia interna da obra) por conter em si uma representação do texto dominante e uma resposta a esta representação no próprio nível da fabulação, resposta esta que passa a ser um padrão de aferição cultural da universalidade tão eficaz quanto os já conhecidos e catalogados. (IDEM, 1982, 23)

A obra teórica de Santiago de uma certa maneira representa uma alternativa ao esquema binário da crítica brasileira dividido entre "conteudismo" e "formalismo". Dialogando com as duas frentes, Santiago tenta escapar de uma ortodoxa concepção de identidade nacional embutida na primeira proposta, como também de uma determinada a-historicidade da segunda.

Daí, talvez, a sua posição estratégica nos Estudos Culturais brasileiros, sendo um dos nomes mais conhecidos da teoria latino-americana contemporânea. A expressão entrelugar passa a ser corrente no domínio da teoria brasileira contemporânea tanto como expressão do cosmopolitismo periférico implicado na pósmodernidade quanto como terminologia especificamente brasileira da teoria póscolonial.

Além do Manifesto Antropofágico, outro texto literário que vai ser fundamental para os Estudos Culturais brasileiros contemporâneos é o conto "A terceira margem do rio" de Guimarães Rosa, retrabalhado, especialmente a partir dos anos 90, como instância para se pensar - Brasil sob a ótica dessa condição de entrelugar, de diferença, de terceiro termo. Uma série de críticos brasileiros e latino-americanistas utiliza o conto para delinear questões teóricas pertinentes à pós-modernidade, ao pós-colonialismo e à própria história brasileira. Essa "terceira margem" apontada por Rosa pode ser um lugar inatingível, do silêncio, da imobilidade ou de uma promessa ainda a ser realizada:

A threshold that we never venture across, and which even now we do not confront. A symbolic prison, so to speak, or the bank of a river, the edge of a sea or cliff, from which bridges are not built and from which we cannot set off back to the interior whence we came, because of the conviction that reality lies neither at the beginning nor at the end, but in the middle of the crossing. It is now an advantage for this restrictive impe-rative of magic to be laid bare, but how many alternatives for the ima-gination are there flourishing on the third bank of the river? (SEVCENKO, 1992, 83)

$\mathrm{Ou}$ ainda o território da problematização do sujeito, alternativa aos binarismos redutores, um entrelugar, um espaço de interpenetrações:

And so the textualizing - fictionally, narratively, historically - of the third term echoes both poetically and e-thically through Latin American, European, Latin American spaces as 
the play of the in-terpenetration of Self in Other in Self extends ever across, ever trans(Atlantically) only as soonto-be unsettled term of provisionality. Play before presence and absence? Before Self, before Other? (McGUIRK, 1996, 195) .

\section{Notas}

1 Ver em Beatriz Sarlo, "Raymond Williams. Uma releitura", Paisagens imaginárias. São Paulo: EDUSP, 1997, pp. 85-95, a discussão mais específica sobre a influência de Raymond Williams no panorama acadêmico argentino desde as décadas de 60 e 70.

2 Na primeira aparição do termo, Santiago utilizava o hifen. Já nos ensaios subseqüentes dos volumes Vale quanto pesa e Nas malhas da letra, ele passa a abolir o hifen de entrelugar.

\section{Referências}

BOSI, Alfredo. Dialética da colonização. São Paulo: Companhia das Letras, 1992.

CANDIDO, Antonio. "Literatura e subdesenvolvimento", A educação pela noite eoutros ensaios. São Paulo: Ática, 1989, pp. 140-162.

HELENA, Lucia Helena. "Queremos a Revolução Caraíba: Identidade cultural econstrução discursiva", Gragoatá 1 (1996), pp. 55-65.

LARSEN, Neil Larsen, Reading North by South. On Latin American LiteratureCulture and Politics. Minneapolis: University of Minnesota Press, 1995.

McGUIRK, Bernard. "Space, Self, Other: Latin America and the 'Third Term' ", Brazil and the Discovery of America. Narrative, History, Fiction. [Bernard McGuirk, Solange Ribeiro de Oliveira, eds.] Lewiston/Queenston/ Lampeter: The Edwin Mellen Press, 1996, pp. 180-198.

MOREIRAS, Alberto. "Elementos de articulación teórica para el subalternismo latinoamericano. Candido y Borges", Revista
Iberoamericana. Vol LXII, 176-177 (1996), pp. 875-891.

ORTIZ, Renato. Cultura brasileira e identidade nacional. São Paulo: Brasiliense,1985.

Cultura e modernidade. São Paulo: Brasiliense, 1991.

A moderna tradição brasileira. Cultura brasileira e indústria cultural. São Paulo: Brasiliense, 1988.

1994.

Mundialização e cultura. São Paulo: Brasiliense,

ROUANET, Sergio Paulo. "A coruja e o sambódromo", Malestar na modernidade. São Paulo: Companhia das Letras, 1993, pp.46-95.

SANTIAGO, Silviano. Uma literatura nos trópicos. São Paulo: Perspectiva, 1978.

Vale quanto pesa. Ensaio sobre questões político-

culturais. Rio de Janeiro: Paz e Terra, 1982.

Letras, 1989.

Nas malhas da letra. São Paulo: Companhia das

SARLO, Beatriz. Paisagens imaginárias. São Paulo: EDUSP, 1997.

SCHWARZ, Roberto. "Nacional por subtração", Que horas são? São Paulo: Companhia das Letras, 1987, pp. 29-48, pp. 47-48.

SEVCENKO, Nicolau. "In Search of the Third Bank of the River: Reflections on the Burden of the Past in Contemporary Brazilian Culture", Travesía. Journal of Latin American Cultural Studies 1:1 (1992), pp. 69-86.

VERONA, Tânia Cristina. "O mundo europeu em Oswald de Andrade", Cultura brasileira. Figuras da alteridade. [Eliana Maria de Melo Souza, ed.] SãoPaulo: Hucitec/FAPESP, 1996, pp. 45-57.

VIEIRA, Else Ribeiro Pires. "Postcolonialisms and the Latin Americas",interventions, Vol 1(2) (1999), pp. 273-281.

"Nudity Versus Royal Robe: Signs in Rotation from (In) Culture to (In)Translation in Latin America", Brazil and the Discovery of America. Narrative, History, Fiction. [Bernard 
McGuirk, Solange Ribeiro de Oliveira, eds.] Lewiston/ Queenston/ Lampeter: The Edwin Mellen Press, 1996, pp.115.

WISNIK, José Miguel. "Comentário", Narrativa: Ficção \& História. [Dirce Côrtes Riedel, ed.] Rio de Janeiro: Imago, 1988, pp. 257-64. 